\title{
Numerical modelling of cathodic protection systems for deep-well casings
}

\author{
A. B. Peratta, J. M. W. Baynham \& R. A. Adey \\ CM BEASY Ltd, UK
}

\begin{abstract}
This work is focused on the 3D simulation of cathodic protection (CP) systems for metallic deep-well casings immersed in stratified soil. The soil is considered as a multi-layered electrolyte with electric conductivity, which varies from layer to layer. Given a CP system, the aim of the simulation is to predict the level of protection (including the normal current entering the structure) against corrosion along the well casing. It is common practice to fill the annular space between soil and metallic structure with cement along the whole depth. The article presents a modelling approach which incorporates this feature. In addition, a unique CP system may be used to protect more than one well. In this case, the simulation is used to analyse problems of interference and load unbalance to the electrical resistance in the power lines and flow lines. The modelling approach is aimed at simplifying the model design, especially for dealing with a multi-layered electrolyte, and improving the accuracy over existing traditional techniques, which entail a detailed and time consuming representation in the model of the interfaces between different layers of the electrolyte. The effects of different scenarios of electrolytes on the CP system are considered.
\end{abstract}

Keywords: cathodic protection, boundary element method, deep-well casings.

\section{Introduction}

The predictive simulation of cathodic protection (CP) systems applied to well casings provides a number of advantages at the design, monitoring and optimization stages of the project. Accurate modelling capabilities help to gain better understanding of the relationship between different $\mathrm{CP}$ variables, the infrastructure to be protected and its surrounding environment. 
An effective method to monitor corrosion in buried structures is to measure the pipe/soil potential along its span. With horizontal pipelines, this is technically feasible; however, the access to data on the local CP performance in well casings is very limited and can only be obtained using expensive logging tools.

Deep-well casing depths vary usually from a few hundred metres to a few kilometres. Along its depth, the casing may penetrate different geological regions with varying physical properties including water content, porosity, permeability and salinity among others. Therefore, from the modelling point of view, the casing can be considered as exposed to a stratified (multi-layered) electrolyte of varying electrical conductivity. Moreover, from layer to layer the polarization properties of the metallic structure may vary in addition to the characteristic conductivity, due to the corrosivity of the medium.

The electrical conductivity of the soil and its distribution are key parameters, which determine how current flows between the anode and the metallic structure, and therefore strongly influence the levels of corrosion protection along the casing. The modelling of CP systems involves predicting the current and potential fields at any point in the electrolyte and at surfaces of electrodes. Under most common situations, this requires solving the steady state charge conservation equation in the electrolyte in $3 \mathrm{D}$ space given by:

$$
\nabla \cdot \mathbf{j}=0, \mathbf{x} \in \Omega
$$

where

$$
\mathbf{j}=-\sigma(\mathbf{x}) \nabla V_{e}(\mathbf{x})
$$

represents current density, $\sigma$ is the electrolyte conductivity, $V_{e}$ is the potential in the electrolyte measured against remote earth, and $\Omega$ represents the integration domain (electrolyte).

The boundary element method (BEM) [1] has been widely used to solve Laplacian equations and in particular simulate cathodic protection systems for underground and offshore structures [2-6]. The most significant advantages of the method are first that the formulation is based on the fundamental solution of the leading partial differential operator in the governing equation and second that it requires only mesh discretisation on the boundaries of the problem. The former aspect confers high accuracy, while the latter substantially simplifies the pre-processing stage of the model, since volume discretisation is not needed. The standard BEM is traditionally aimed at solving homogeneous electrolytes. In the case of non-homogeneous conditions, it is common practice to combine BEM with domain decomposition or multi-region (MR) technique. In this way the electrolyte, considered as piecewise homogeneous, is represented as a collection of sub-regions, each one of them with homogeneous conductivity. Then, neighbouring regions are connected to each other by prescribing continuity of potential and normal current density through their common interface [3].

In this contribution, a new technique for computing accurately and efficiently 3D problems involving deep-well casings immersed in multi-layered soil is used and compared against the traditional MR. The goal of the multi-layer (ML) approach is to avoid including in the model the interfaces between different layers, by employing a fundamental solution specifically designed for multi-layer materials. The major advantages over the more traditional MR approach are 
mainly the reduction in the engineering time to prepare a model, since interfaces do not need to be included in the model. Also the solving time is minimised, since only the degrees of freedom representing the well casing and the anodes need to be considered. An important consequence of this feature is that the number of layers will not significantly affect the computational cost of the calculation. Moreover, a common limitation in BEM is that the distance between two elements of the mesh must not be too small in comparison to the characteristic length of the largest element, otherwise accuracy and stability of the solution is compromised. This translates into a practical limitation in modelling thin layers of electrolyte, or models in which the thickness of the layer is small in comparison with its lateral extension. However, the ML approach does not suffer from this limitation, and therefore allows the end user to include thin layers without major problems of accuracy or high computational cost.

The article is organised as follows: section 2 provides briefly some background information on the ML. Section 3 presents a comparison between MR and ML results in a particular case scenario. Section 4 demonstrates the modelling capabilities of subsurface thin layers. This type of modelling is difficult and time consuming with the standard MR approach but rather straightforward to conduct with the ML. Section 5 presents a method for modelling the cement in the annular space between the outer tube and soil. Section 6 presents the case of a single anodic bed protecting two distant casings. Overall conclusions are given in section 7 .

\section{Multi-layer modelling approach}

The modelling of $\mathrm{CP}$ systems protecting well casings is intrinsically three dimensional (3D). Hence 3D BEM kernels are required. The ML approach incorporates the stratified nature of the medium into the corresponding Green's function. Then BEM is applied in the same way as in the case of the homogeneous electrolyte, except that the Green's function for the homogeneous Laplace equation given by

$$
G\left(\mathbf{x}_{i}, \mathbf{x}_{j}\right)=\frac{1}{4 \pi r}
$$

is replaced by the multi-layer Green's function given by:

$$
G\left(\mathbf{x}_{i}, \mathbf{x}_{j}, m, n\right)=\frac{1}{4 \pi \sigma_{m}} \sum_{k=1}^{N_{a p p r}} \frac{\alpha_{i j m n}}{\left\|\mathbf{x}_{i}-\mathbf{x}_{j}+\mathbf{g}_{i j m n}\right\|}
$$

where $\mathbf{x}$ denotes the 3D position vector, the sub-indices $i$ and $j$ stand for the source and field point, respectively; $r=\left\|\mathbf{x}_{j}-\mathbf{x}_{i}\right\| ; m$ and $n$ indicate the layer of the source and field points, respectively; $a_{i j m n}$ is a weight coefficient and $\mathbf{g}_{i j m n}$ denotes a displacement vector. The summation is performed over the number of approximating terms $N_{a p p r}$ in the multi-layer Green's function. The calculation of the weight and displacement vectors goes beyond the scope of this contribution and can be found in refs. [2, 3, 7-9] and their citations. Finally, any standard BEM strategy can be employed. In this case, collocation direct BEM has been adopted. 


\section{Case study: comparison between MR and ML}

A number of comparison tests and validations of the ML approach have been performed using as reference the MR technique. This section introduces a conceptual model used to compare the results and calculation performance of both methods. The conceptual model consists of a single well casing and one ICCP anodic bed in stratified soil consisting of 7 layers. The well is $1750 \mathrm{~m}$ deep and consists of 4 sections of different diameter. The specification of the main metallic tube relevant to the simulation is shown in Table 1 where $z$ is the depth co-ordinate, $D_{\text {ext }}$ the tube outside diameter, $t$ its wall thickness and $R$ its electrical resistance.

Table 1: Main tube specifications.

\begin{tabular}{|c|c|c|c|c|c|c|}
\hline Section & $\begin{array}{c}\text { Soil } \\
\text { layer }\end{array}$ & $z_{\min }$ & $z_{\max }$ & $D_{\text {ext }}$ & $t$ & $R$ \\
\hline & & $\mathrm{m}$ & $\mathrm{m}$ & $\mathrm{m}$ & $\mathrm{m}$ & Ohm $/ \mathrm{m}$ \\
\hline 1 & 1 & 0 & 300 & 0.35 & 0.012 & $1.37 \times 10^{-5}$ \\
\hline 2 & 2 & 300 & 500 & 0.25 & 0.012 & $1.94 \times 10^{-5}$ \\
\hline 3 & $2,3,4$ & 500 & 1200 & 0.175 & 0.012 & $2.83 \times 10^{-5}$ \\
\hline 4 & $4,5,6,7$ & 1200 & 1750 & 0.15 & 0.012 & $3.34 \times 10^{-5}$ \\
\hline
\end{tabular}

Fig. 1 (right) illustrates the conceptual model. The numbers on the left-hand side indicate the conductivity of each layer, while the scale on the right-hand side indicates the $z$ coordinate of each interface. The dot circle labelled as " 0 " in the figure represents the origin of the $x y z$ system of coordinates assumed in this work. The anode bed, represented by a $30-\mathrm{cm}$ diameter by $8 \mathrm{~m}$ long cylinder, is located $75 \mathrm{~m}$ apart from the well in $x$ direction.

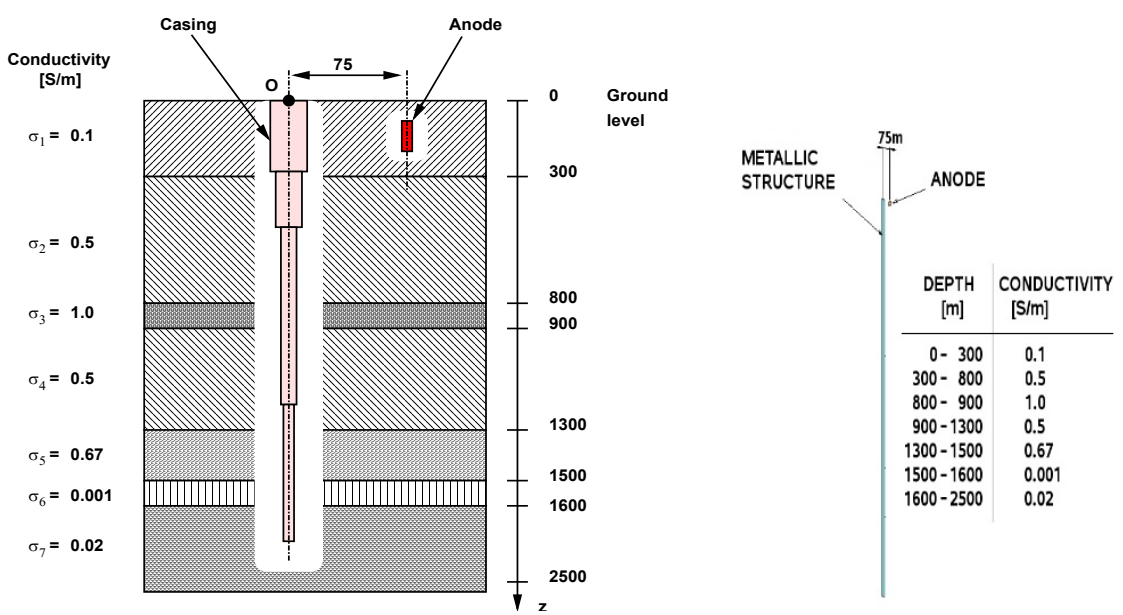

Figure 1: Conceptual model of MR (left). ML model (right). 
The size of the linear system of equations that needs to be solved in this case would be $N_{D O F} \times N_{D O F}=3339 \times 3339$, where $N_{D O F}$ is the number of degrees of freedom. The top of the anode is at $30 \mathrm{~m}$ deep and the total current injected into the CP system is $I_{A}=10 \mathrm{~A}$. The scenario posed for this case study is similar to the one presented in earlier works apart from minor variations. The discretisation mesh of the MR model is shown in Fig 2. The interfaces between layers need to be discretised and incorporated in the model. On the other hand, the ML requires the discretisation of the casing and anode only, thus avoiding elements on the interfaces and bounding box.

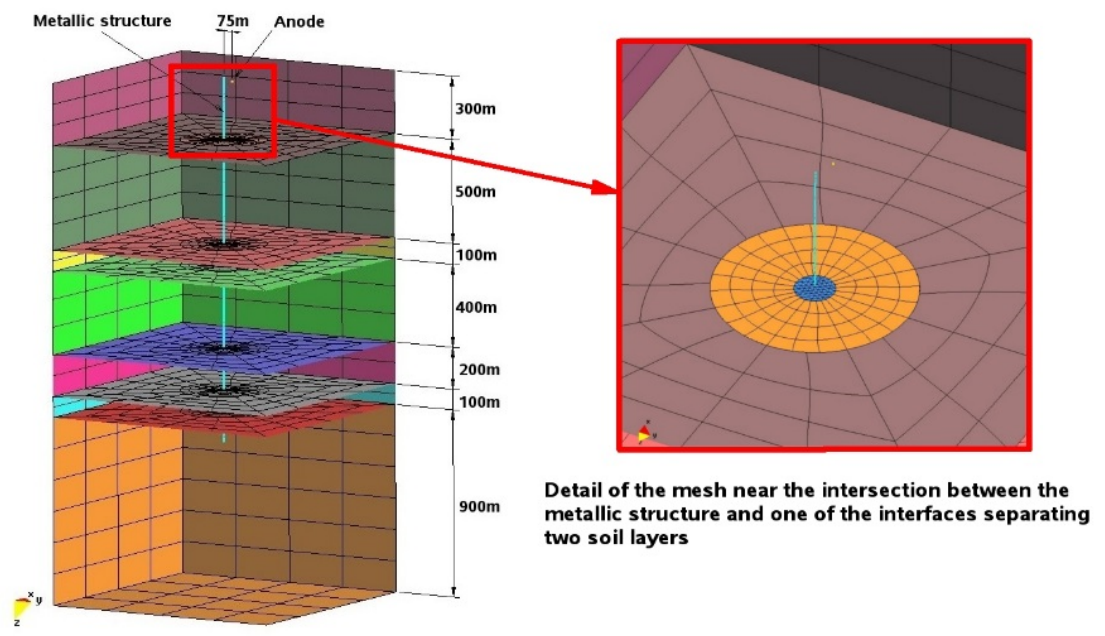

Figure 2: Mesh discretisation for the MR model.

In this example the casing was discretised with 177 tube elements and the anode with 10 elements. Hence, the total number of degrees of freedom is 187 and the corresponding linear system of equations is of size $187 \times 187$. The ML model is shown in Fig. 1 (right). A comparison between MR and ML for the over potential along the structures is shown in Fig 3. More detailed results of the comparison can be found in Peratta et al. [3].

\section{Effect of thin layers}

One of the major advantages of the ML is that it is not limited with regard to the number of layers or horizontal extent or thickness of each layer. In order to illustrate this feature and to provide better understanding of the effects of the conductivity of thin layers on the over potential distribution on the whole structure, an uppermost subsurface layer $30 \mathrm{~m}$ thick with variable conductivity has been added to the model. This type of study is more difficult to conduct with the MR approach in view of the fine discretisation needed for the interface of the thin layer. 
The over potential as function of depth for different sub-surface conductivities in layer 0 is shown on the left of Fig. 4 . It can be seen that the conductivity of the soil in the first layer does not substantially affect the over potential anywhere else apart from the first $30 \mathrm{~m}$ in contact with that layer. Each curve corresponds to a different soil conductivity in layer 0 which extends from ground level to $30 \mathrm{~m}$. The depth is represented in logarithmic scale, since the main changes of over potential occur in the first few soil layers starting from ground level.

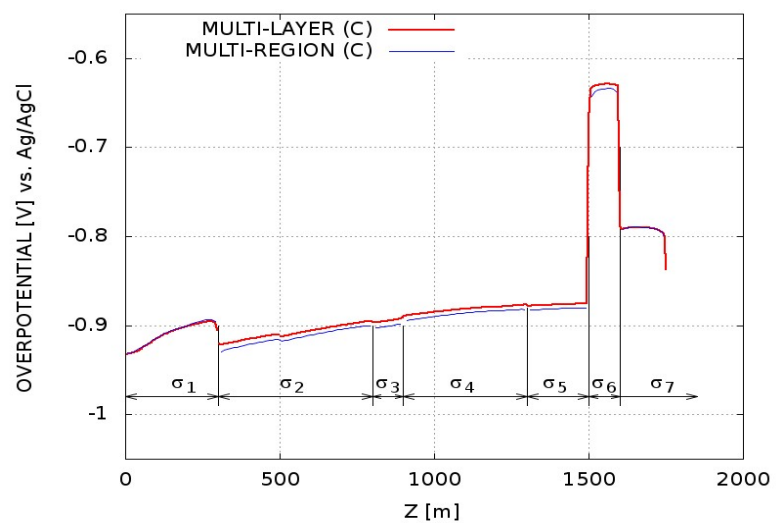

Figure 3: Over potential along depth. Comparison between ML (thick red) and MR (thin blue).

Fig. 4 on the right shows the average over potential in the region $0<z<30 \mathrm{~m}$ of the casing for different electrolyte conductivity of the first layer. Lower conductivity in the subsurface layer translates into more negative over potentials.
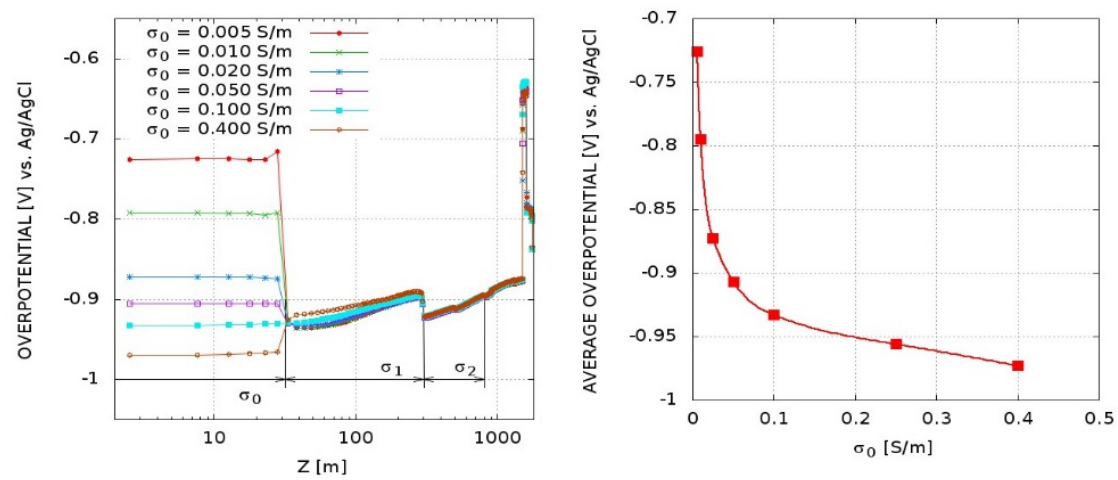

Figure 4: Over potential along the well casing in the 8-layer soil model. 


\section{Cement layer}

This section considers the influence of a cement layer surrounding the casing from the electrical conductivity point of view, i.e. any other effect apart from the ohmic drop has not been considered.

Fig. 5 (left) shows an arbitrary cross section of the well under consideration. The current density in the concrete is considered to be oriented in the radial direction. Under these circumstances, the concrete is introduced into the model as a passive medium which introduces an additional ohmic-type voltage drop in the electrolyte. The idea is to derive the equivalent resistance offered by the concrete and use it to adjust the original polarization curve of the steel, as illustrated in Fig. 5 (right). The original polarization curve of the steel shown in red is corrected with a linear ohmic drop (dashed line). The resulting polarization curve used in the model is represented by a thick black continuous line. Assuming that the current density vector $j$ has only radial component, its magnitude at the internal radius $r_{2}$ varies linearly with the voltage drop across the cement according to:

$$
j\left(r_{2}\right)=k_{c} r_{2} \log \left(r_{1} / r_{2}\right) \Delta \phi,
$$

where $\Delta \phi=\phi\left(r_{1}\right)-\phi\left(r_{2}\right)$ is the potential difference across the cement and $k_{c}$ the cement electrical conductivity. For practical convenience, the surface distribution of ohmic resistance $\rho_{s}$ is defined as

$$
\rho_{s}=\left(r_{2} / k_{c}\right) \log \left(r_{1} / r_{2}\right)
$$

Then the over potential $V$ of the equivalent polarization contemplating the $I R$ drop across the concrete is corrected according to:

$$
V=V-\rho_{s} j .
$$
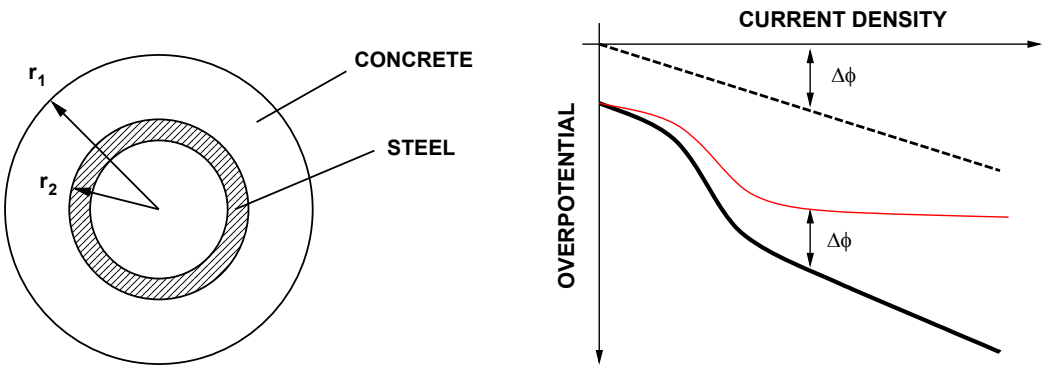

Figure 5: Cross-section of the well casing (left). Adjustment of the original polarization curve due to the ohmic drop in the cement (right). 
In order to investigate the effect of the concrete surrounding the metallic case, the same model of well casing described above was considered in the following scenarios named "BARE", "WET", "DRY" and "MIX".

- "BARE": This scenario considers the case of bare steel (no concrete at all).

- "WET": The metallic case is inside a cement cylinder of external diameter $0.9 \mathrm{~m}$. The concrete is considered highly porous and water has infiltrated.

- "DRY": Same as wet, but with lower conductivity.

- "MIX1": This scenario assumes that the pipe sections 1,2 and 3 are inside a cylindrical column of dry cement, while section $4(1200<z<1750 \mathrm{~m})$ is considered to be damaged and therefore with the conditions of the wet case.

The two types of concrete considered are shown in Table 2.

Table 2: Cement conductivity.

\begin{tabular}{|c|c|}
\hline Type of concrete & Electric conductivity \\
\hline Wet & $0.025 \mathrm{~S} / \mathrm{m}$ \\
\hline Dry & $0.004 \mathrm{~S} / \mathrm{m}$ \\
\hline
\end{tabular}

The details of each case scenario are listed in Table 3 and the relevant polarisation curves used are shown in Fig. 6.

Table 3: Case scenarios.

\begin{tabular}{|c|c|c|c|c|c|c|c|c|}
\hline \multirow{2}{*}{ Section } & \multicolumn{2}{|c|}{ Span } & \multirow{2}{*}{$r_{1}$} & \multirow{2}{*}{$r_{2}$} & & \multicolumn{3}{|c|}{$\rho_{s}$} \\
\cline { 2 - 6 } \cline { 6 - 9 } & $\mathrm{z}_{\min }$ & $z_{\max }$ & & & $r_{1}-r_{2}$ & $\mathrm{WET}$ & $\mathrm{DRY}$ & $\mathrm{MIX1}$ \\
\hline & $\mathrm{m}$ & $\mathrm{m}$ & $\mathrm{m}$ & $\mathrm{m}$ & $\mathrm{m}$ & $\mathrm{Ohm} \cdot \mathrm{m}^{2}$ & $\mathrm{Ohm} \cdot \mathrm{m}^{2}$ & $\mathrm{Ohm} \cdot \mathrm{m}^{2}$ \\
\hline 1 & 0 & 300 & 0.45 & 0.175 & 0.05 & 2.87 & 17.95 & 17.95 \\
\hline 2 & 300 & 500 & 0.45 & 0.125 & 0.1 & 2.78 & 17.38 & 17.38 \\
\hline 3 & 500 & 800 & 0.45 & 0.0875 & 0.1375 & 2.49 & 15.56 & 15.56 \\
\hline 4 & 1200 & 1750 & 0.45 & 0.075 & 0.15 & 2.33 & 14.59 & 2.33 \\
\hline
\end{tabular}

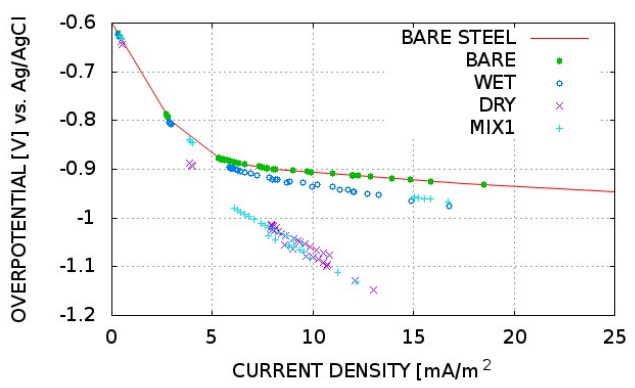

Figure 6: Polarisation curves. 
The external diameter of the cement lining is kept constant and equal to $0.45 \mathrm{~m}$, hence the thickness of the cement wall $h$ varies along depth.

As a consequence, the resulting polarization curve is modified with different $I R$ drop resistances depending on the sector of the well considered.

Fig. 7 shows a comparison of normal current density, over potential, metal voltage and axial current density for the different scenarios.

These results illustrate how the performance of the CP system is affected by the electric resistivity of the cement layer, which depends on a variety of other physical and chemical properties. In particular, the change of resistivity due to porosity and water saturation has significant effect on the distribution of overpotential along the well casing.
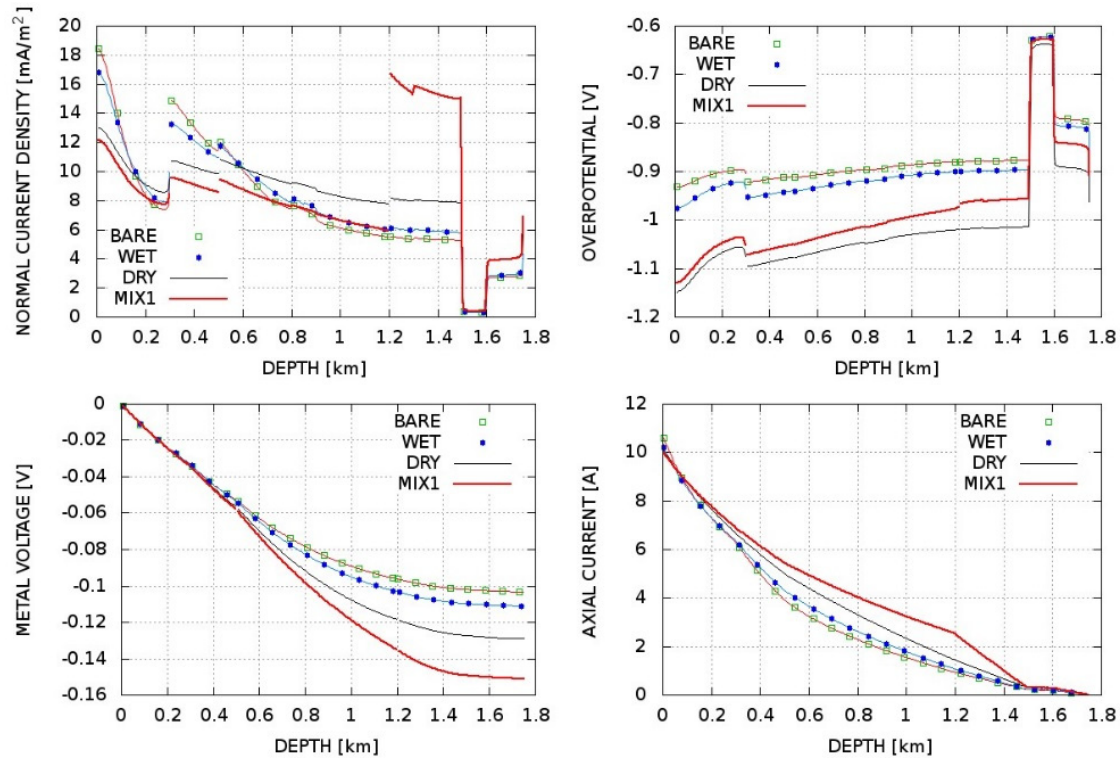

Figure 7: Normal current density (top left), over potential (top right), metal voltage (bottom left) and axial current density (bottom right) in function of depth for "bare", "wet", "dry" and "mix" scenarios.

\section{Multiple wells}

The physical situation of this case scenario is shown in Fig 8. The model corresponds to the bare steel case and includes the $I R$ drop in the connections to the power supply but not those in the associated flow lines. The two wells are protected with a single ICCP anode. 


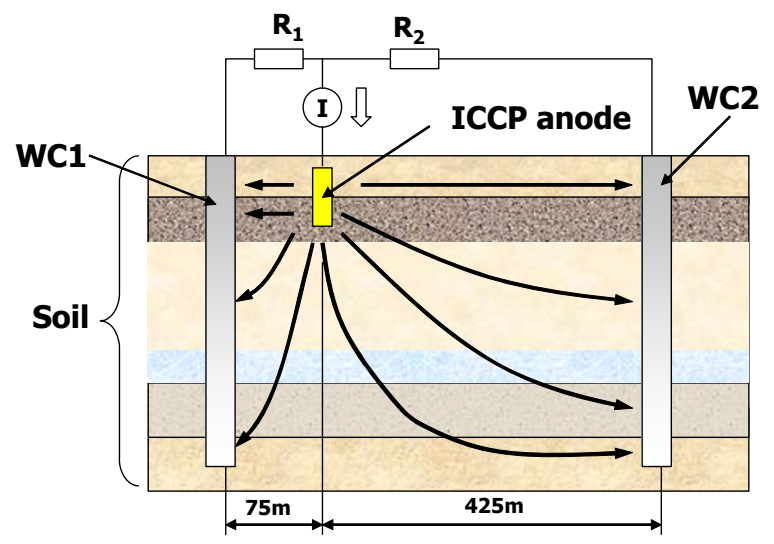

Figure 8: Two well casings connected to a unique ICCP anode.

The anode is $75 \mathrm{~m}$ apart from $\mathrm{WC} 1$ and $425 \mathrm{~m}$ apart from $\mathrm{WC} 2$. The electrical resistance of the power supply cable is represented with the resistors R1 and R2 of 0.4 and $1.44 \mathrm{Ohm}$, respectively. Therefore, the unbalance in the currents flowing to the two wells is not only due to the different resistance in the return path circuit but also due to the ohmic $I R$ drop in the soil.

Fig. 9 shows the results of normal current density, over potential, metal voltage and axial current flowing along each well casing.
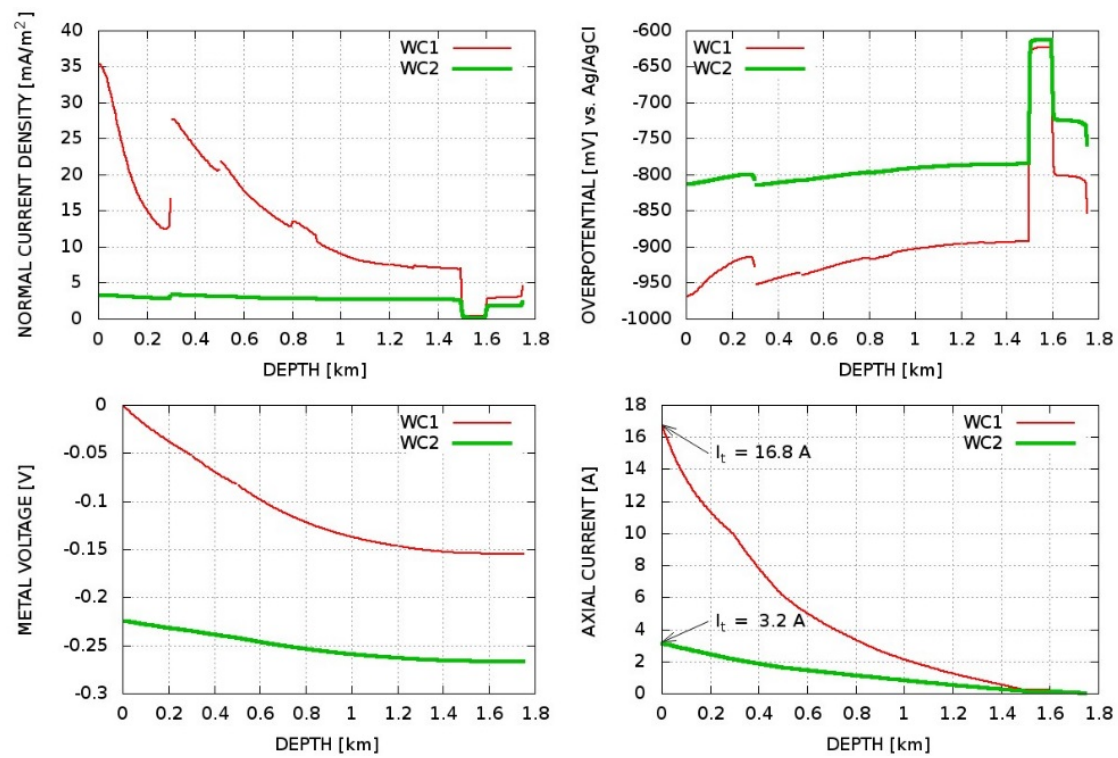

Figure 9: Comparison of normal current density, overpotential, metal voltage and total axial current for the two well casings connected to the ICCP anode. 
As expected, the normal current density on the well casing more distant from the anode (WC2) is significantly lower in comparison to the one closer (WC1). In addition, the over potential on the former is on average approximately $100 \mathrm{mV}$ lower than on the latter, except in the less conductive soil layer at depth between 1500 and $1600 \mathrm{~m}$ where the difference in over potential is negligible.

In this case, none of the well casings show anodic behaviour but with different conditions this may occur and would be predicted by the model.

\section{Conclusions}

A new approach for the numerical modelling of CP systems applied to well casing structures in vertically stratified soil has been developed. A significant advantage over the traditional multi-region technique is the reduction of the number of degrees of freedom. In addition, the modelling preparation is simplified, as interfaces between soil layers do not need to be modelled at all.

The multilayer (ML) approach can easily represent thin layers of electrolyte without additional mesh discretisation thus substantially reducing the work of the user.

A feature of the ML is that the computational cost of the calculation does not increase with the number of layers. The results obtained with the ML are in good agreement with the traditional multi-domain approach.

The most important benefit of the ML approach is the simplicity in its application to well casing applications as the data can be prepared very easily and the calculations made within a few minutes. Consequently, sensitivity studies on proposed new designs can be quickly completed.

The model can be used to optimise the use of measurement techniques such as surface $E-\log I$ measurements and Corrosion and Protection Evaluation Tool (CPET) logs to improve the cost effectiveness and reliability of CP designs [10].

An approach for incorporating the cement that fills the annular space between the metal and the soil has been proposed and the influence of different qualities of the cement on the CP performance has been quantified. In cases where there is doubt about the quality of the cement in a particular layer, the model can be used to assess its impact and identify effective corrosion control options.

The model has been applied to multiple well situations where there is the possibility of interference between the wells. Results have been presented where not only the ohmic drop in the electrolyte is considered but also the $I R$ drop in the power supply cables, flow lines and other associated equipment and structures is modelled.

\section{References}

[1] Brebbia, C.A., Telles, J.C.F. \& Wrobel, L.C., Boundary Element Techniques - Theory and Application in Engineering, Springer Verlag: Berlin, Heidelberg, NY, Tokyo, 1984.

[2] Peratta, A.B., Baynham, J.M.W. \& Adey, R.A., A computational approach for assessing coating performance in cathodically protected transmission 
pipelines. NACE Corrosion 2009 Conference, Atlanta, Georgia, Paper 6595, 2009.

[3] Peratta, A.B., Baynham, J.M.W. \& Adey, R.A., Advances in cathodic protection modelling of deep well casings in multi-layered media. NACE Corrosion 2009 Conference, Atlanta, Georgia, Paper 6555, 2009.

[4] Riemer, D.P. \& Orazem, M.E., Modelling coating flaws with non-linear polarization curves for long pipelines. Advances in Boundary Elements, vol. 12: Corrosion and Cathodic Protection Modelling and Simulation, ed. R.A. Adey, WIT press: Southampton, pp. 225-259, 2005.

[5] Riemer, D.P. \& Orazem, M.E., Application of boundary element models to predict the effectiveness of coupons for accessing cathodic protection of buried structures. Corrosion, 56, pp. 794-800, 2000.

[6] Adey, R.A. \& Baynham, J., Design and optimization of cathodic protection systems using computer simulation. Corrosion 2000, NACE-00723, NACE International, Orlando, Florida, 2000.

[7] Seremet, V.D., Handbook of Green's Functions and Matrices, WIT Press: Southampton, Boston, 2002.

[8] Ymeri, H., Nauwelaers, B. \& Maex, K., Computation of conductance and capacitance for IC interconnects on a general lossy multilayer substrate. Active and Passive Electronic Components, 24, pp. 87-114, 2001.

[9] Smedes, T., van der Mejis, N.P. \& van Gendered, A.J., Boundary element methods for 3D capacitance and substrate resistance calculations in inhomogeneous media in a VLSI layout verification package. Advances in Engineering Software, 20, pp. 19-27, 1994.

[10] Roche, M., Vittonato, J. \& Jebara, M., Cathodic protection modelling of deep wells casing by 3D software simulation: Comparison with E-Logl and CPET data. NACE Corrosion 2008, Paper 08273, 2008. 